$\begin{array}{cc}\text { PRAMANA } & \text { (c) Indian Academy of Sciences } \\ \begin{array}{c}\text { journal of } \\ \text { physics }\end{array} & \begin{array}{l}\text { Vol. xx, No. } x \\ \text { xxxx xxxx } \\ \text { pp. } 1-9\end{array}\end{array}$

\title{
Dilepton production in nucleon-nucleon collisions around 1 GeV/nucleon: a theoretical update
}

\author{
R. Shyam ${ }^{1,2}$, and U. Mosel ${ }^{1}$ \\ ${ }^{1}$ Institut für Theoretische Physik, Universität Giessen, D-35392 Giessen, Germany \\ ${ }^{2}$ Saha Institute of Nuclear Physics, 1/AF Bidhan Nagar, Kolkata 700064, India
}

\begin{abstract}
We present a fully relativistic and gauge invariant framework for calculating the cross sections of dilepton production in nucleon-nucleon $(N N)$ collisions which is based on the mesonexchange approximation for the $N N$ scattering amplitudes. Predictions of our model are compared with those of other covariant models that have been used to describe this reaction. We discuss the comparison of our calculations with the old DLS and the recent HADES data.
\end{abstract}

Keywords. Dilepton production, NN collisions, effective Lagrangian model

PACS Nos 25.75.Dw, 13.30.Ce, 12.40.Yx

\section{Introduction}

Experiments with High Acceptance Di-electron Spectrometer (HADES) are aimed at searching for medium modifications of hadrons at moderately high temperatures $(T<100$ $\mathrm{MeV}$ ) and baryonic densities up to 3 times the normal nuclear matter density. Due to negligible final state interactions with surrounding medium, dileptons $\left(e^{+} e^{-}\right)$provide a very clean and powerful probe for this purpose.

A recurring feature of the dilepton spectra measured in nucleus-nucleus $(A A)$ collisions has been the enhancement (above known sources) in the invariant mass distribution of the cross sections in the intermediate region of dilepton invariant masses $(M)$. This has been the case for experiments performed for bombarding energies ranging from as low as $1.0 \mathrm{GeV} /$ nucleon (DLS and HADES data [1,2]), through the SPS energies (40 $158 \mathrm{GeV} /$ nucleon) [3-5] to the energies employed by the PHENIX collaboration at RHIC (which correspond to invariant mass of $200 \mathrm{GeV} /$ nucleon) [6]. The enhancement seen at the SPS energies are understood in terms of the modification of the $\rho$ meson spectral function in the hadronic medium [7].

The large dileptons yields observed in the DLS experiment (even for the light collision systems ${ }^{12} \mathrm{C}+{ }^{12} \mathrm{C}$ ) in the invariant mass range of $0.2-0.5 \mathrm{GeV}$, are yet to be explained satisfactorily [8-12]. Independent transport model calculations (TMC) have been unable to describe these data fully even after including contributions from (i) the decay of $\rho$ and $\omega$ mesons which are produced directly from the nucleon-nucleon $(N N)$ and pion-nucleon scattering in the early reaction phase [13], (ii) the in-medium $\rho$ spectral functions [14], (iii) the dropping $\rho$ mass with corresponding modification in the resonance properties [8], (iv) 
an alternative scenario of the in-medium effect - a possible decoherence between the intermediate meson states in the vector resonance decay [11]. This led to term this discrepancy as "DLS-puzzle" $[8,13]$ which persists even now. The new dilepton production data of the HADES Collaboration agree remarkably well with the corresponding DLS data [15]. Therefore, there is no longer any question against the validity of the DLS data and the failures to explain them by various transport models have to do with problems in the theoretical calculations.

Cross sections for dilepton production in elementary nucleon-nucleon $(N N)$ collisions are important inputs in the TMC. For the $N N$ bremsstrahlung processes most TMC use the cross sections predicted by a semiclassical soft photon approximation (SPA) model [16,17]. An important point made in a recent TMC [18], is that if one scales up the SPA cross sections for the $p p$ and $p n$ systems by factors of 2-4, the observed dilepton yields of both DLS and HADES experiments can be reproduced. These enhancement factors are motivated by the larger elementary $N N$ dilepton cross sections predicted by the calculations reported in Refs. $[19,20]$. However, these large cross sections are not in agreement with those reported in several previous studies [21-23]. They also do not agree with results of a more recent study of the dilepton production in $N N$ collisions [24]. Therefore, the puzzle seems to have reduced to a proper understanding of the dilepton production in elementary $N N$ collisions.

In the next section we present a brief comparison of various models used to calculate the dilepton yields in elementary $N N$ collisions. It is important to understand the differences seen in the predictions of various models in order to have a proper theoretical description of the new HADES data on the dilepton production not only in elementary $p p$ and $p n$ collisions but also in the $A A$ collisions.

\section{Comparison of various models of dilepton production in elementary $N N$ Collisions}

Calculations reported in Refs. [19,20] (to be referred as KK), and [23] (to be referred as SM1) use the same basic model to calculate the dilepton production in $N N$ collisions. Both KK and SM1 account for the initial interaction between two incoming nucleons by an effective Lagrangian which is based on the exchange of the $\pi, \rho, \omega$ and $\sigma$ mesons. The coupling constants and the form factors at the nucleon-nucleon-meson vertices are the same in both studies. These parameters were determined in Ref. [21] by directly fitting the $T$ matrices of the $N N$ scattering in the relevant energy region, and they have been used in the successful descriptions of the $N N \rightarrow N N \pi$ [25], $p p \rightarrow p \Lambda K^{+}, p p \rightarrow p \Sigma^{0} K^{+}$[26,27] and $N N \rightarrow N N \eta[28]$ reactions.

The major difference between calculations of Refs. [19] and [23] lies in the method of implementing the gauge invariance of the $N N$ bremsstrahlung amplitudes. To investigate this issue, the dilepton production cross sections within the SM1 model have been recalculated recently [24] (to be referred as SM2) by using a pseudoscalar (PS) coupling

$$
\mathcal{L}_{N N \pi}=i g_{N N \pi} \bar{\Psi}_{N} \gamma_{5} \boldsymbol{\tau} \cdot \boldsymbol{\Phi}_{\pi} \Psi_{N},
$$

for the nucleon-nucleon-pion $(N N \pi)$ vertex while the KK model employs a pseudovector (PV) coupling

$$
\mathcal{L}_{N N \pi}=-\frac{g_{N N \pi}}{2 m_{N}} \bar{\Psi}_{N} \gamma_{5} \gamma_{\mu} \boldsymbol{\tau} \cdot\left(\partial^{\mu} \boldsymbol{\Phi}_{\pi}\right) \Psi_{N}
$$



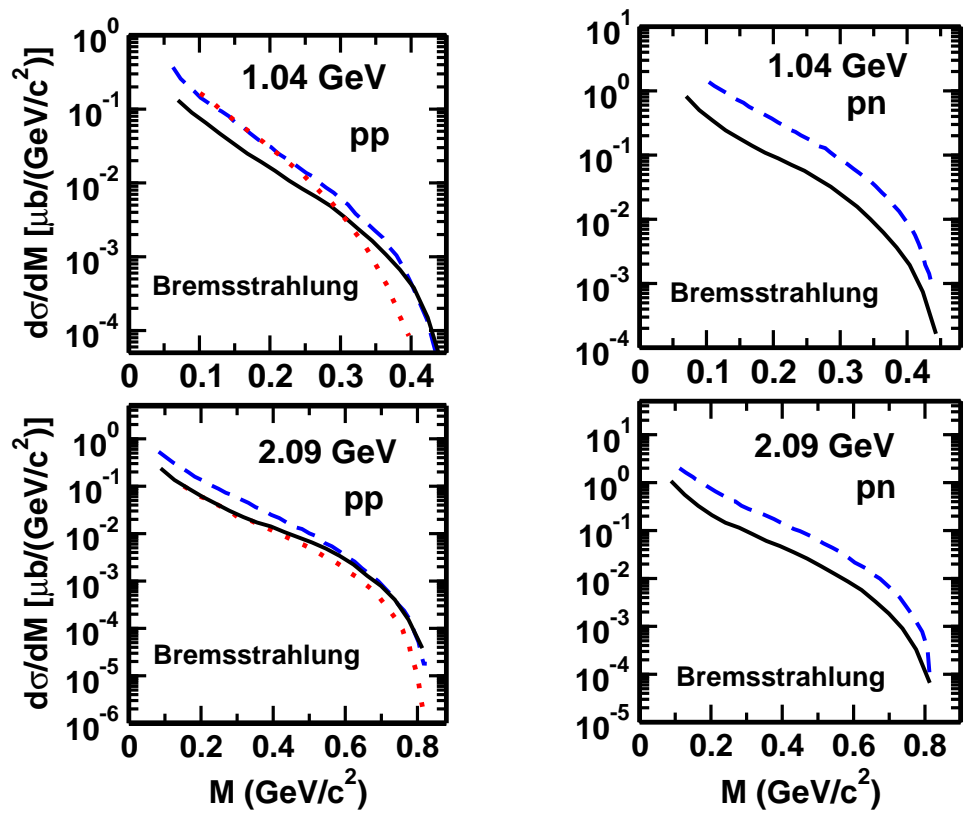

Figure 1. The invariant mass distribution of the $N N$ bremsstrahlung contributions to the dilepton spectra in $p p$ (left) and $p n$ (right) collisions at the beam energies of 1.04 $\mathrm{GeV}$ and $2.09 \mathrm{GeV}$. Results obtained within our model are shown by solid lines while those of Refs. [22] and [19] by dotted and dashed lines, respectively.

for this vertex. With a PV vertex an extra term (known as contact or seagull term) appears in the model which comes from the electromagnetic coupling (by the substitution $\partial^{\mu} \rightarrow \partial^{\mu}-i e m A^{\mu}$, where $m$ is $+1,0,-1$ for positive, neutral and negative pions) in the $N N \pi$ Lagrangian. On the other hand, with a PS vertex, the theory is free from this term. For point-like particles both prescriptions lead to gauge invariant $N N$ bremsstrahlung amplitudes. However, nucleons have finite sizes - to account for this fact form factors are introduced at the corresponding vertices. Form factors are also required to quench the unphysical contributions from higher momenta. In the presence of form factors the gauge invariance may be violated. As is shown in Ref. [21], in case of the PS coupling it is straight forward to introduce the form factors while retaining the gauge invariance by following the method of Ref. [29]. However, with contact terms present in the theory in the PV case, the introduction of form factors without violating the gauge invariance, is a more delicate issue. There are several prescriptions for this and various procedures may lead to quite different results (see, e.g., [30]. Therefore, the use of the PS coupling for the $N N \pi$ vertex makes the dominant pion exchange contributions free from such ambiguities.

In Ref. [22] (to be referred as dJM model), instead of the one-boson exchange picture of SM1, SM2 and KK calculations, the nucleon-nucleon interaction is included via $T$ matrices that are based on the Paris potential. This model provides a reliable description of the off-shell behavior of the effective $N N$ interaction. In addition, the rescattering terms are included in both nucleon and $\Delta$ intermediate states - these terms are not present in both 


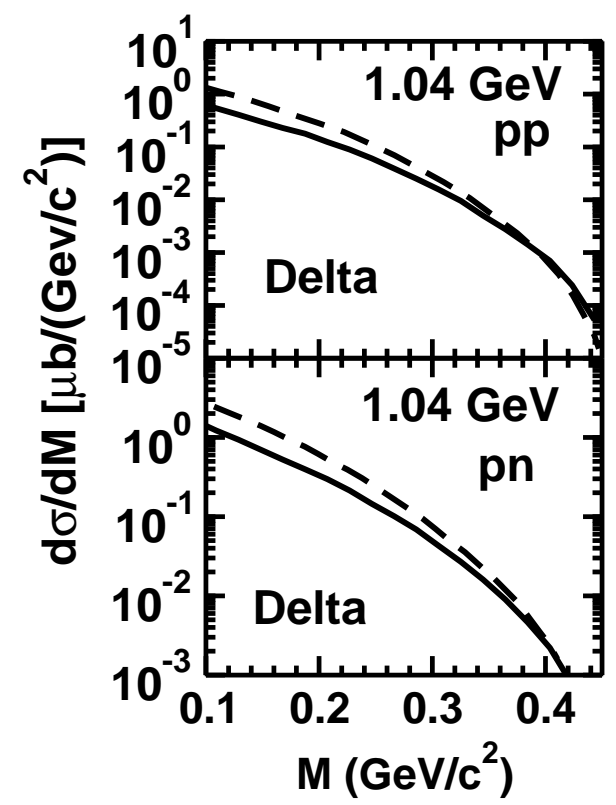

Figure 2. The invariant mass distribution of the $\Delta$ isobar contribution to the dilepton spectra in $p p$ (upper panel) and $p n$ (lower panel) collisions the beam energy of 1.04 $\mathrm{GeV}$. The results of our model are shown by full lines while those of Ref. [19] by dashed line.

SM $(1 \& 2)$ and KK calculations. Furthermore, the degeneracy of the PS and PV choices for the pion coupling is resolved to a large extent in this model; one has to simply use different parameters to obtain the same fit. However, the price paid here is that the nucleon current is not gauge invariant. These authors rectify this problem in an ad-hoc manner.

In the next section we present a comparison of the predictions of various models for the invariant mass distributions of the dileptons produced in $N N$ collisions.

\section{Comparison of cross sections calculated within various model}

In Fig. 1, we show the invariant mass distribution of the $p p$ and $p n$ bremsstrahlung contributions to the dilepton spectra at beam energies of 1.04 and $2.09 \mathrm{GeV}$ as calculated in SM2 model (solid lines). Also shown here are the results for this reaction as reported in Refs. [22,19]. We first discuss the results for the $p p$ reaction shown in the left panel of this figure. At the outset we remark that the SM2 cross sections are very similar to those reported in Refs. [21] and [23]. However, some differences are seen between the cross sections of SM2 and dJM models and also between them and the KK calculations. Whereas the dJM cross sections are larger than those of SM2 in the mass region $M<0.25 \mathrm{GeV}$, they are almost identical to the latter at $2.09 \mathrm{GeV}$ in this mass region. At both the beam energies, dJM results are smaller than SM2 ones for $M>0.25 \mathrm{GeV}$. In contrast to this, the KK cross sections are larger than those of SM2 everywhere for $M<0.6 \mathrm{GeV}$. An 


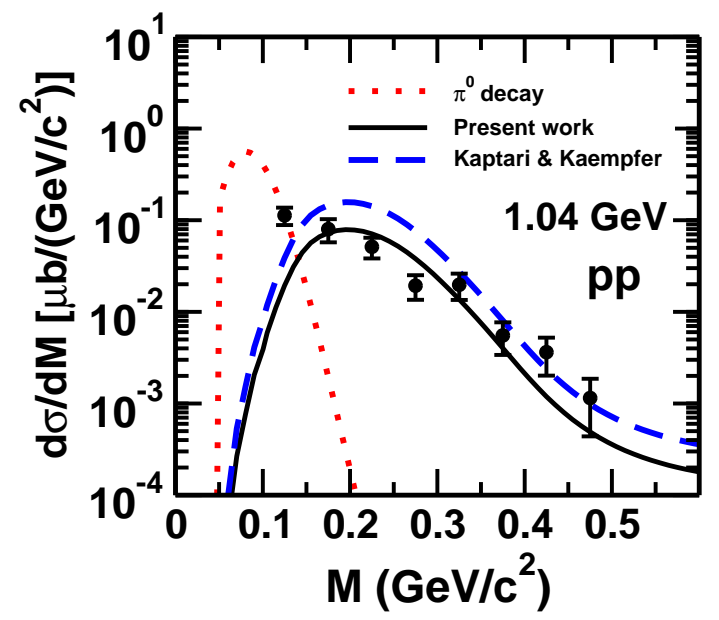

Figure 3. The calculated dilepton invariant mass distribution for the $p p$ collision at the beam energy of $1.04 \mathrm{GeV}$ in comparison to the DLS data. The contribution of the $\pi^{0}$ Dalitz decay is also shown here which is the same as that in Ref. [23].

important point to note is that there is no overall multiplicative factor that differentiates the results of various models.

It is rather surprising that despite using the same diagrams, input parameters and gauge invariance restoration procedure, the SM2 $p p$ bremsstrahlung cross sections are lower than those of Ref. [19]. Of course, in Ref. [19] a pseudovector $N N \pi$ vertex has been used as compared to the pseudoscalar one employed in SM2. In this context, it is worthwhile to note that for the real photon production, the covariant model calculations do not depend on the choice of the $N N \pi$ coupling (PS or PV) as is shown in Ref. [31]. In case of dileptons, different results can arise for two couplings from the magnetic part of the $N N \gamma$ vertex. In fact, in Ref. [32] it is shown that $p p$ dilepton bremsstrahlung contributions obtained with the PV $N N \pi$ coupling are actually smaller than those calculated with the PS one at the beam energy of $2 \mathrm{GeV}$. The calculations presented in Ref. [23] also support this to some extent.

In the right panel of Fig. 1, we compare the SM2 results with those of the KK model for the $p n$ collisions. In this case, the situation is even more contrasting - the SM 2 cross sections are a factor of 3-4 lower than the KK ones. The results of the dJM model are not available for this reaction. It is highly desirable that this discrepancy between the results of the two models for the $p n$ case is properly understood.

In Fig. 2, we show a comparison of SM2 and KK results for the invariant mass distribution of the $\Delta$ isobar contribution to the dilepton production in $p p$ and $p n$ collisions at the beam energy of $1.04 \mathrm{GeV}$. We note that here too the KK cross sections are larger than SM2 by factors of $\sim 2$ at smaller values of $\mathrm{M}$ even though the two models have used the same ingredients and input parameters for this part and there is no ambiguity related to gauge invariance as the resonance vertex is gauge invariant by its very construction.

In Fig. 3, we compare the SM2 and KK total cross sections for the dilepton production in $p p$ collisions with the DLS data at the beam energy of $1.04 \mathrm{GeV}$. The cross sections 


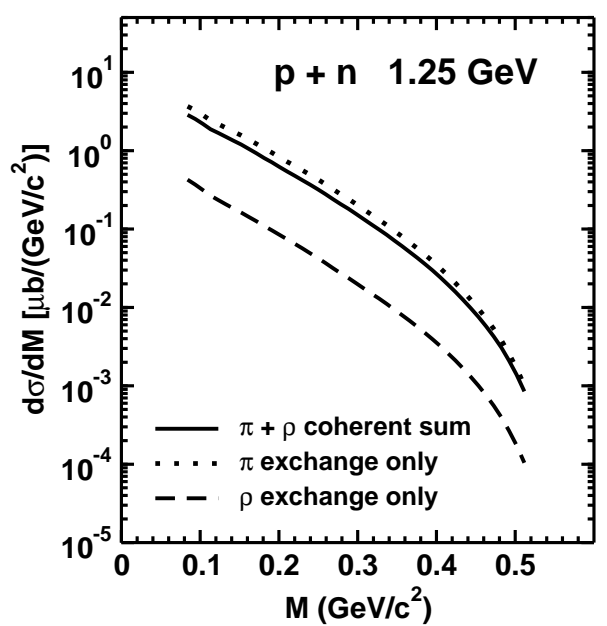

Figure 4. Contributions of $\pi$ (dotted line) and $\rho$ (dashed line) exchange diagrams to the invariant mass distribution of the dileptons produced in proton-neutron collisions at the beam energy of $1.25 \mathrm{GeV}$. Their coherent sum is shown by the solid line.

calculated within SM2 model are folded with appropriate experimental filter and final mass resolution. The folded KK cross sections have been obtained by assuming that the folding procedure does not affect the ratios of the unfolded cross sections in the two cases. In this figure we have also shown cross sections for the $\pi^{0}$ Dalitz decay $\left(\pi^{0} \rightarrow \gamma e^{+} e^{-}\right)$ which are the same as those in Ref. [23]. It is seen that KK cross sections overestimate the DLS data for $M<0.3 \mathrm{GeV}$ where statistical errors are smaller. The data have larger error bars for $M>0.3 \mathrm{GeV}$. In this context, the HADES data on the elementary dilepton production reactions are useful because of their low statistical error. A comparison of the HADES dilepton mass spectra measured in the $p p$ and quasi-free $n p$ reactions [33] and the predictions of the SM2 and KK models, has been reported in Ref. [34]. There one notices that for the case of $p p$ reaction, once again the predictions of the SM2 model are in pretty good agreement with the data while the KK model overestimates them.

In Fig. 4, we examine the role of various meson exchange processes to the dilepton production in the $p n$ collisions at $1.25 \mathrm{GeV}$ beam energy. In this figure, the dotted and dashed lines show the individual contributions of the $\pi$ - and $\rho$-exchange graphs to the dilepton invariant mass distribution. Contributions of other meson exchange processes are relatively smaller. The coherent sum of the two terms is shown by the solid line. It is clear that the $\pi$-exchange process dominates the cross sections and more significantly, it interferes destructively with the $\rho$-exchange term as the coherent sum of the two diagrams is even smaller than the pion-only exchange term. This implies that a larger $\rho$-exchange term [20] will lead to even smaller total cross sections. 

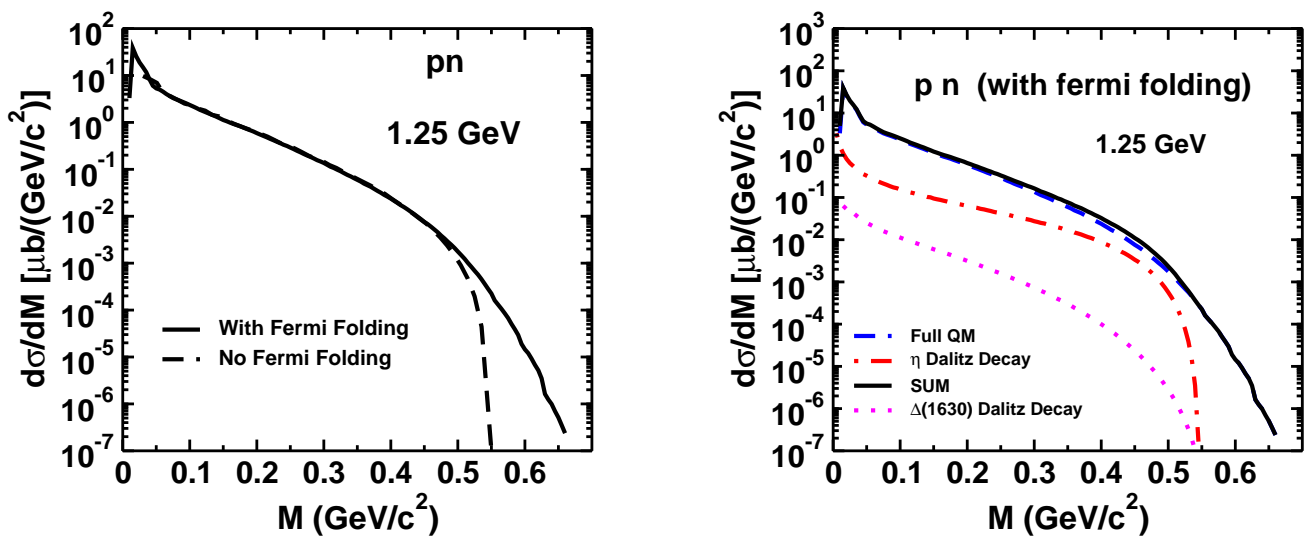

Figure 5. (Left panel) the dilepton invariant mass distribution in $n p$ (dashed line) and quasi-free $n p$ interaction (solid line) at $1.25 \mathrm{GeV} /$ nucleon. In the later case the center mass energy is smeared by including the neutron momentum distribution in the deuteron using the Argonne V18 potential. (Right panel) the dilepton invariant mass distribution in the quasi-free $n p$ collisions at $1.25 \mathrm{GeV} /$ nucleon calculated within the model of Ref. [24] (dashed line). Also shown are the contributions of $\eta$ meson and $\Delta(1600)$ Dalitz decay processes by dashed-dotted and dotted lines, respectively. Their incoherent sum is shown by the solid line.

\subsection{Calculations at the HADES energies}

In the experiments carried out by the HADES Collaboration, proton and deuteron beams with kinetic energies of $1.25 \mathrm{GeV} /$ nucleon were incident on a proton target. In this measurement the quasi-free $n p$ reactions were selected by detection of fast spectator protons from the deuterium breakup in the forward direction.

As has already been stated above, the HADES data on the dilepton invariant mass distribution (DIMD) in the $p p$ reaction were described well by the SM2 model [33,34] while the KK model overestimated them. However, for the quasi-free $n p$ collisions, both models fail to reproduce the experimental cross sections. The shape of the DIMD changes dramatically when going from $p p$ to $p n$ interactions. One notices that (1) in the dilepton invariant mass region of 0.15 to $0.35 \mathrm{GeV}$, the yield is about 9 times higher in the $n p$ case as compared to that of the $p p$ case, (2) in the quasi-free $n p$ case, the tail of the DIM extends to much larger values of $M$, (3) the ratio of quasi-free $n p$ to $p p$ cross sections reaches almost a value of 100 at $M=0.5 \mathrm{GeV}$.

To understand points (1)-(3), we introduce the following 3 additional features in the SM2 model for the $n p$ case. (i) The available energy in the center of mass is smeared to include the neutron momentum distribution in the deuteron using the Argonne V18 potential [35]. The consequence of this procedure is that the $d p$ reaction results is a smeared $n p$ reaction with center of mass energies in excess of the threshold for the $\eta$ meson production (see, e.g., Ref. [36]). (ii) Hence, the contribution from the $\eta$ Dalitz decay is taken into account. In these calculations the total cross sections for the $n+p \rightarrow n+p+\eta$ reaction is taken from the Ref. [28] where a good description is achieved of the available corresponding 
experimental data. (iii) The dilepton yields from the Dalitz decay of higher mass delta resonances are included. Our preliminary results are shown in Fig. 5. It was already noted in Ref. [34] that the predictions of the SM2 model are in agreement with the $n p$ data for $M<0.3 \mathrm{GeV}$. In the left panel of Fig. 5, we note that the Fermi folding procedure leads to an extended tail in the invariant mass distribution. In the right panel, we show the total cross sections obtained by Fermi folding the cross sections of the SM2 model together with contributions of the $\eta$ meson and $\Delta(1600)$ isobar Dalitz decay processes. Their incoherent sum is shown by the solid line. It is seen that the inclusion of contributions (ii) and (iii) leads to only a marginal enhancement in the summed cross sections over those of the SM2 model for $M$ values around $0.50 \mathrm{GeV}$. Therefore, a proper understanding of point (3) is still lacking at this stage.

\section{Summary and Conclusions}

In summary, we have shown that the dilepton yields from the $N N$ bremsstrahlung process calculated within the SM $(1 \& 2)$ models $[23,24]$ are lower than those of the KK model $[19,20]$ by factors of 2-4 for both $p p$ and $p n$ collisions at $1-2 \mathrm{GeV}$ incident energies. The recent HADES measurements have confirmed that the cross sections calculated within the SM2 model are in good agreement with the experimental data for $p p$ reaction while those of the KK model overestimate them. This was also the case for the DLS data as shown in Fig. 3. This implies that care must be exercised in using the larger $N N$ bremsstrahlung cross sections in the transport model calculations in order to explain the DLS and HADES data obtained in the nucleus-nucleus collisions.

With HADES collaboration confirming the old DLS data, the resolution of the "DLS" puzzle has indeed now shifted to a better understanding of the theoretical models. In this context, the focus should be to achieve a proper explanation of the HADES data for dilepton production in quasi-free $n p$ interaction. We showed that smearing of the center of mass energy to include the neutron momentum distribution in deuteron, does lead to extended tails in the dilepton invariant mass distributions. However, the absolute magnitudes of the cross sections in the region of dilepton invariant masses around $0.5 \mathrm{GeV}$ are still not explained even after taking into account the contributions from the Dalitz decays of $\eta$ meson and higher mass resonances. Work is in progress to resolve this new puzzle.

\section{5. acknowledgments}

This work is supported by the Helmholtz International Center for FAIR (HIC for FAIR) under the LOWE program.

\section{References}

[1] R. J. Porter et al., Phys. Rev. Lett. 79, 1229 (1997).

[2] G.Agakichiev et al., Eur. Phys. J. A 41 (2009) 243.

[3] G. Agakichiev et al., Eur. Phys. J. C 41, 475 (2005).

[4] D. Adamova et al., Phys. Rev. Lett. 91, 042301 (2003).

[5] R. Arnaldi et al., Phys. Rev. Lett. 96, 162302 (2006). 
[6] S. Afanasiev et al., arXiv:0706.303 [nucl-ex].

[7] R. Rapp, J. Phys. G 34, S405 (2007); R. Rapp, Nucl. Phys. A782, 275 (2007); H. van Hees and R. Rapp, Phys. Rev. Lett. 97, 102301 (2006); J. Ruppert, C. Gale, T. Renk, P. Lichard, and J. I. Kapusta, Phys. Rev. Lett. 100, 162301 (2008).

[8] C. Ernst, S. A. Bass, M. Belkacem, H. Stöcker, and W. Greiner, Phys. Rev. C 58, 447 (1998).

[9] W. Cassing and E. L. Bratkovskaya, Phys. Rep. 308, 65 (1999).

[10] R. Rapp and J. Wambach, Adv. Nucl. Phys. 25, 1 (2000).

[11] K. Shekhter, C. Fuchs, A. Faessler, M. Krivoruchenko, and B. Martemyanov, Phys. Rev. C 68, 014904 (2003).

[12] G. Agakichiev et al., Phys. Rev. Lett. 98, 052302 (2007).

[13] E. L. Bratkovskaya, W. Cassing, R. Rapp, and J. Wambach, Nucl. Phys. A634, 168 (1998).

[14] W. Cassing, E. L. Bratkovskaya, R. Rapp and J. Wambach, Phys. Rev. C 57, 916 (1998).

[15] G. Agakishiev et al., Phys. Lett. B663, 43 (2008).

[16] R. Rückl, Phys. Lett. B64, 39 (1976).

[17] C. Gale and J. Kapusta, Phys. Rev. C 35, 2107 (1987);ibid, Phys. Rev. C 40, 2397 (1989).

[18] E. L. Bratkovskaya and W. Cassing, Nucl. Phys. A807, 214 (2008).

[19] L. Kaptari and B. Kämpfer, Nucl. Phys. A764, 338 (2006).

[20] L. Kaptari and B. Kämpfer, Phys. Rev. C 80, 064003 (2009)

[21] M. Schäfer, H. C. Dönges, A. Engel and U. Mosel, Nucl. Phys. A 575, 429 (1994)

[22] F. de Jong and U. Mosel, Phys. Lett. B379, 45 (1996).

[23] R. Shyam and U. Mosel, Phys. Rev. C 67, 065202 (2003).

[24] R. Shyam and U. Mosel, Phys. Rev. C 79, 035203 (2009).

[25] R. Shyam and U. Mosel, Phys. Lett. B426, 1 (1998).

[26] R. Shyam, Phys. Rev. C 60, 055213 (1999).

[27] R. Shyam, G. Penner and U. Mosel, Phys. Rev. C 63, 022202(R) (2001).

[28] R. Shyam, Phys. Rev. C 75,055201 (2007).

[29] D. O. Riska, Prog. Part. Nucl. Phys. 11, 199 (1984).

[30] A. Usov and O. Scholten, Phys. Rev. C 72, 025205 (2005).

[31] M. Schäfer, T. S. Biro, W. Cassing, U. Mosel, H. Nifenecker and J. A. Pinston, Z. Phys. A 339, 391 (1991).

[32] F. de Jong and U. Mosel, Phys. Lett. B392, 273 (1997).

[33] G. Agakishiev et al., arXiv:0910.5875 [nucl-ex].

[34] B. Ramstein et al., arXiv:0912.2677 [nucl-ex].

[35] R. B. Wiringa, V. G. J. Stoks, and R. Schiavilla, Phys. Rev. C 51, 38 (1995).

[36] I. Fröhlich et al., arXiv:0909.5373 